\title{
La gestión turística sostenible de los establecimientos de alojamiento de la ciudad de Cuenca Ecuador: Ámbito Empresarial
}

\section{Sustainable tourism management of accommodation establishments in the city of Cuenca Ecuador: Business Area}

\author{
Marcela Alexandra Galarza Torrres ${ }^{1 *}$ \\ ${ }^{1}$ Universidad de Cuenca \\ *alexandra.galarza@ucuenca.edu.ec
}

DOI: https://doi.org/10.26871/killkana_social.v2i3.322

\begin{abstract}
Resumen
La aplicación de buenas prácticas sostenibles ha ido generando en los últimos años un mayor interés en el sector turístico ecuatoriano; este hecho se evidencia en la gran cantidad de establecimientos de alojamiento certificados, hecho que resulta por un lado de los beneficios que las empresas reciben tras finalizar un proceso de certificación, así como en la creciente conciencia sostenible que actualmente presentan los turistas al momento de elegir un establecimiento de alojamiento. Sin embargo esta situación no ocurre en la ciudad de Cuenca, pues existe un bajo interés en el empresario hotelero cuencano por incurrir en procesos de certificación sostenible. Desde esta perspectiva este trabajo investigativo, tuvo como objetivo determinar el nivel de aplicación de buenas prácticas en establecimientos de alojamiento de la ciudad de Cuenca; enfocado de forma específica en el aspecto empresarial. El estudio tuvo un enfoque cualitativo con alcance descriptivo mediante el desarrollo de encuestas cerradas a través de muestreo, empleando la metodología de la Norma Smart Voyager donde participaron 7 establecimientos de alojamiento. Los resultados obtenidos indican que existe una fuerte debilidad en la estructura empresarial hotelera, y un casi nulo manejo de la percepción de la calidad enfocada en la atención y el servicio al cliente, así como muy poco conocimiento sobre temas relacionados con la sostenibilidad. De los resultados obtenidos se deriva el hecho que no existen en la actualidad instrumentos que posibiliten un incremento en la gestión turística sostenible de establecimientos de alojamiento en la ciudad de Cuenca, o beneficios que permitan incrementar la participación de estos establecimientos en la aplicación de buenas prácticas sostenibles.
\end{abstract}

Palabras clave: Sostenibilidad, Gestión, Calidad, Establecimientos Alojamiento, Recursos Humanos, Buenas Prácticas de Turismo Sostenible.

\begin{abstract}
The application of good sustainable practices has generated in the last years a greater interest in the Ecuadorian tourist sector; this fact is evident in the large number of certified accommodation establishments, a fact that results on the one hand from the benefits that companies receive after completing a certification process, as well as the growing sustainable awareness that tourists present at the moment of choosing an accommodation establishment. However, this situation does not occur in the city of Cuenca, because there is a low interest in the hotel business in Cuenca for incurring sustainable certification processes. From this perspective, this research work aimed to determine the level of application of good practices in housing establishments in the city of Cuenca; Focused specifically on the business aspect. The study had a qualitative approach with descriptive scope through the development of closed surveys through sampling, using the methodology of the Smart Voyager Standard where 7 accommodation establishments participated. The results indicate that there is a strong weakness in the hotel business structure, and almost no management of the perception of quality focused on customer service and attention, as well as very little knowledge on issues related to sustainability. From the results obtained, it is derived that there are currently no instruments that allow an increase in sustainable tourism management of accommodation establishments in the city of Cuenca, or benefits that allow increasing the participation of these establishments in the application of good practices sustainable.
\end{abstract}

Key words: Sustainability, Management, Quality, Accommodation Establishments, Human Resources, Good practices of Sustainable Tourism. 


\section{Introducción}

Los impactos de la actividad turística en el medio ambiente resultan evidentes hoy en día para cualquier persona; así, la planificación de esta actividad para minimizar dichos impactos en el entorno se presenta como incuestionable. Sin embargo, no siempre ha sido de esta manera. Durante muchos años, el desarrollo turístico tuvo una escasa planificación y consciencia de sus efectos sobre el medio ambiente. Las normas reguladoras del sector turístico, ya de por sí escuetas, no hacían referencia alguna al desarrollo turístico, a no ser que destruyera el entorno, y no existía una conciencia ambiental entre los promotores turísticos ni entre los consumidores. (De las Heras, 2008)

El desarrollo sostenible o sostenibilidad, son términos que ya se ha incorporado a la vida actual, aunque aún no a todos los diccionarios. Aparece por primera vez en el panorama internacional a través de la denominada "Estrategia Mundial para la Conservación" en 1980. En este documento, que fue elaborado por una organización conservacionista denominada la Unión Internacional para la Conservación de la Naturaleza (UICN); que hoy conserva esas mismas siglas pero cuyo nombre ha sido modificado por el de "Unión Mundial para la Naturaleza", aparecía el término "desarrollo sostenido".

Este término ha sido adoptado en la industria turística como un modelo de gestión turística más responsable, equitativo, ético y justo, así este sector ha implementado varias herramientas encaminadas a una búsqueda más sostenible de este sector, indistintamente de su área de especificación (turismo comunitario, ecoturismo, etc).

Dentro del turismo, el sector hotelero es quizá el sector que mayores retos está enfrentando actualmente en el ámbito de la sostenibilidad, esto, debido principalmente a las nuevas exigencias de un mercado que cada vez prefiere un mayor compromiso con el ambiente. Desde el uso correcto de los recursos naturales como el agua, hasta un correcto aprovisionamiento y almacenamiento de la materia prima, el sector alojamiento debe considerar una exhaustiva y minuciosa operación, que permita no solamente ser más amigable con el ambiente y las comunidades locales, sino también optimizar sus recursos para obtener así una mayor rentabilidad. (Pulido Fernández, 2006)

En el ámbito de la sostenibilidad, las buenas prácticas para un turismo sostenible constituyen en la actualidad una importante herramienta que permite a los establecimientos de alojamiento no únicamente consolidar una operación más sostenible, sino también que cada departamento cuente con procesos técnicos y estandarizados que busquen alcanzar los objetivos planteados, buscando siempre la satisfacción de una demanda más participativa y decisiva frente a un desarrollo sostenible.

En el caso del Ecuador, varias son las instituciones no gubernamentales (mayoritariamente) que otorgan certificaciones de turismo sostenible, entre ellas está Rainforest Alliance, Servqual, etc. Ecuador cuenta con más de cien establecimientos certificados en esta área, hecho que refleja un cambio en los modelos de gestión hotelera tradicionales.

La ciudad de Cuenca por su parte está considerada como el segundo destino turístico más visitado de la sierra ecuatoriana y el cuarto a nivel país; cuenta con características muy apetecibles para el desarrollo turístico, sin embargo no existen todavía establecimientos certificados en sostenibilidad. (Fundación Municipal de Turismo para Cuenca y Ministerio de Turismo, 2011)

\section{Marco Teorico}

El turismo acorde a su definición técnica se encuentra muy relacionado con los desplazamientos de las personas a lugares distintos a su residencia habitual quienes acorde al tiempo de estancia pueden diferenciarse en visitantes excursionistas, residentes y no residentes (Organización Mundial del Turismo, 2013); sin embargo en los últimos años y a pesar de las crisis económicas que muchos países han experimentado muchos países, se ha mantenido de manera creciente y se ha posicionado como uno de los sectores más importantes de la economía internacional. De acuerdo a la Organización Mundial del Turismo (Organización Mundial del Turismo, 2017), el turismo aporta con el 10\% del Producto Interno Bruto (PIB) mundial, generando exportaciones por más de 1.5 billones de dólares, constituyendo el $7 \%$ del comercio internacional. (Santiago Romero y Alvarez, 2017).

En el caso de Ecuador, se estima que este país es el que presenta mayor flujo turístico receptivo y estimación de la demanda internacional (Ayaviri, Quispe, y Sánchez, 2017). Así también Ecuador ha logrado un crecimiento constante en el volumen de arre los internacionales, superando el millón y medio de visitantes, con una tasa de ocupación promedio superior al $50 \%$, con ingresos que ascienden a 1075.5 millones de USD al año 2016 (Santiago Romero y Alvarez, 2017); este hecho refleja un saldo muy positivo para nuestro país y de manera especial a la ciudad de Cuenca, destino que ha incrementado sus visitas tras ser declarada como Patrimonio Cultural de la Humanidad por la UNESCO en 1999.

Bajo esta perspectiva, se puede observar que el turismo se consolida cada vez más como un fenómeno social y cultural, que tiene efectos directos en la economía de los lugares visitados (Organización Mundial del Turismo, 2013), su impacto puede también analizarse a través de tres diferentes ámbitos: económico, social y ambiental, que constituyen los tres elementos claves para un desarrollo sostenible.

El crecimiento y diversificación de la industria del turismo ha dado lugar a la aparición de nuevas modalidades turísticas, destinos y alojamientos que persiguen atraer a los viajeros y turistas con una oferta atractiva, competitiva en precio, calidad y con experiencias enriquecedoras de su estilo de vida; (Pulido Fernández, 2015), sin embargo la conciencia ambiental de los viajeros y clientes de hoteles los hace valorar muy positivamente las buenas prácticas 
ambientales y la gestión sostenible de los recursos naturales.

Turismo sostenible es el que atiende las necesidades actuales de los turistas y de los destinos, al tiempo que protege y fomenta las oportunidades para el futuro (Organización Mundial del Turismo, 2013); por lo tanto se consolida como una vía hacia una gestión más integral de todos los recursos que permita compatibilizar el desarrollo económico y social de la industria y los destinos con las expectativas estéticas y de experiencia de los turistas, respetando la integridad cultural y el entorno ambiental del área visitada. (Ayuso, 2008)

Tour Operators Initiative (2013) indica que la sostenibilidad ya no es una tendencia, sino un atributo de valor diferencial y decisivo para los viajeros de todo el mundo; pues el $50 \%$ de los viajeros mundiales optan por alojarse en establecimientos más ecológicos.

Acorde a TripAdvisor Leader Survey (TripAdvisor, 2013) el $79 \%$ de los viajeros a nivel mundial declara que las prácticas eco sostenibles en los alojamientos turísticos son importantes, en consonancia con esta afirmación el $91 \%$ de los establecimientos de alojamiento turístico consideran que mantener una gestión turística sostenible es importante pero tan solo el $77 \%$ ha implementado medidas relacionadas con el desarrollo sostenible. Por otra parte son los hoteles de América del Norte y América del Sur quienes más suelen conceder gran importancia a la aplicación de medidas ecológicas. (Campodónico, 2016)

Otra de las cifras que realmente destacan en materia de sostenibilidad asociada con los viajes y el turismo, indican que el $34 \%$ de los clientes pagaría más por un hotel responsable y un $71 \%$ de los encuestados está planeando viajes a destinos más sostenibles (Trip Advisor Barometer, 2013).

Ante estos datos es evidente que en la industria del turismo la Innovación tecnológica, la imagen de marca y por ende el turismo sostenible, marcarán la hoja de ruta del turismo y la hotelería durante los próximos años, pues estas cuestiones constituyen la clave de la experiencia turística del futuro y son ya muy valorados hoy por los clientes y huéspedes más allá del destino, le precio, la ubicación y los servicios. (Boullón, 2006)(Boullón, 2006).

Aunque la industria hotelera no se encuentre entre las que generan un mayor impacto ambiental visible sobre el entorno, esto no quiere decir que no sea necesario su compromiso con el medio ambiente y la incorporación de prácticas ambientales respetuosas, buscando la implicación de sus clientes como parte fundamental del proceso de prestación del servicio. Por otro lado, se trata de una actividad que afecta a muchas personas de los municipios receptores y estas buenas prácticas contribuirán a mejorar la integración de los establecimientos en el territorio y las relaciones con sus vecinos. (Hoteles Sostenibles, 2016)

Para la empresa, el desarrollo sostenible significa la creación de riqueza, a la vez que disminuye el impacto ambiental de su actividad. El aumento de la ecoeficiencia es la forma principal de contribución de las empresas al desarrollo sostenible y, cada vez más, se considera un elemento de primer orden en el diseño de estrategias empresariales.

Toda empresa moderna debería incorporar hoy en día los criterios de protección del medio ambiente, esenciales para la gestión general del negocio dentro de una política general de responsabilidad social corporativa y asumir la parte de responsabilidad que le corresponde en la conservación y mejora del medio ambiente. Una empresa comprometida con el medio ambiente es una empresa comprometida con una sociedad cada vez más consciente de los temas ambientales y que empieza a exigir a los agentes económicos una actuación contundente en este sentido. (Pulido Fernández, 2005)

\section{Metodología Aplicada}

La metodología empleada en el presente estudio tiene enfoque cualitativo de alcance descriptivo, para lo cual se ha llevado auditorías exhaustivas mediante con los estudiantes de la carrera de Hotelería de la Facultad de Ciencias de la Hospitalidad de la Universidad de Cuenca durante seis meses consecutivos, con la intensión de levantar toda la información concerniente a diez establecimientos de alojamiento de distinta categoría de la ciudad de Cuenca. Para ello se tomó como referencia los seis criterios del ámbito empresarial que establece la Guía de Buenas Prácticas para Turismo Sostenible de Rainforest Alliance (entidad que ha certificado a más de cincuenta establecimientos de alojamiento de todo el país).

Esta guía está basada en la norma "Smart Voyager" la cual incluye una serie de criterios que tienen como base la consideración estricta de la legislación nacional (Constitución de la República del Ecuador, Ley de Turismo, Ley de Gestión Ambiental, Ley de Prevención y Control de la Contaminación Ambiental, Ley de Defensa del Consumidor, Ley de Seguridad Social, Código del Trabajo, Norma Técnica de Ecoturismo, Instructivo para el Registro de Centros Turísticos Comunitarios y otras normas vigentes relacionadas con establecimientos de alojamiento vigentes en el país. (Smart Voyager, 2016), así como parámetros internacionales reconocidos en el país, los cuales han sido empleados para la valoración de las operaciones turísticas de hospedaje y servicios de alimentos y bebidas.

La norma Smart Voyager emplea una metodología con una base técnica que mantiene la rigurosidad y operatividad de sus criterios, los cuales a su vez obedecen a distintos aspectos relacionados al personal, la gestión de la calidad, la seguridad, la gestión financiera contable, la gestión de la sostenibilidad, y comunicación y mercadeo. En este marco la norma está desarrollada con una metodología con base técnica que mantiene la rigurosidad de sus criterios y la operativa de los mismos en diferentes escenarios, sin que ello implique una modificación en su escala de medición particular.

Los establecimientos de hospedaje seleccionados para la presente investigación se clasificaron en: hoteles, 
pensiones y hostales. Respecto a los establecimientos de hospedaje, la toma de información fue con un muestreo al azar, acorde al porcentaje de ocupación que estos establecimientos reflejaron en el año 2017 al Ministerio de Turismo.

La información fue procesada a través de los formularios establecidos por la organización Rainforest Alliance, para los programas de certificación en turismo sostenible "Smart Voyager", esta metodología ha permitido establecer correctamente el estudio de las variables propuestas, que en este caso son los seis criterios delimitados para el ámbito empresarial del mencionado programa.

\section{Resultados}

Los establecimientos investigados fueron seleccionados acorde al nivel de ocupación que mantuvieron en el año 2017 acorde a su categoría. Así se ha seleccionado una pensión de lujo (Mansión Alcázar), tres hoteles de primera (Carvallo, Azhir 360, Crespo) una hostal de primera (Casa Lloret) Una total de segunda categoría (Macondo) y un hotel de segunda categoría (Rio Piedra)

\subsection{Gestión de la Sostenibilidad: Política de sostenibili- dad}

Constituye el conjunto de lineamientos económicos, sociales y ambientales que permitan minimizar los impactos negativos y maximizar los positivos de su gestión, para con ello conseguir sus objetivos y trabajar de manera sostenible. (Rainfores Alliance, 2004). Este criterio abarca tres grandes componentes que se constituyen a su vez en requisitos mínimos que establece la norma para su cumplimiento, estos son: Misión y Visión enfocadas a una gestión sostenible, política de sostenibilidad y una planificación de la gestión sostenible de la empresa turística.

Tabla 1 Criterios que establece la Norma Smart Voyager para la Gestión de Sostenibilidad

\begin{tabular}{|c|c|c|c|}
\hline Criterio & Subcriterio & Evidencias requeridas & Evaluación \\
\hline $\begin{array}{c}\text { Política de } \\
\text { sostenibilidad }\end{array}$ & $\begin{array}{c}\text { Plan } \\
\text { estratégico de } \\
\text { la empresa } \\
\text { Políticas }\end{array}$ & $\begin{array}{c}\text { - Misión y visión em- } \\
\text { Valorial definidos de } \\
\text { la empresa }\end{array}$ & $50 \%$ \\
\hline empresariales & $\begin{array}{c}\text { Manuales de } \\
\text { puestos de } \\
\text { trabajo }\end{array}$ & $\begin{array}{c}\text { - Normas y reglas de } \\
\text { operación por cada } \\
\text { departamento. } \\
\text { Normas y reglas para } \\
\text { cada proceso y pro- } \\
\text { cedimiento. }\end{array}$ & $25 \%$ \\
\hline Planificación & $\begin{array}{c}\text { Planes de } \\
\text { accion para } \\
\text { cada area de la } \\
\text { empresa }\end{array}$ & $\begin{array}{c}\text { - Cronograma de pla- } \\
\text { nificación estratégica }\end{array}$ & $25 \%$ \\
\hline
\end{tabular}

Fuente: Guía de Buenas Prácticas para Turismo Sostenible
Tabla 2 Política de Sostenibilidad en establecimientos de alojamiento de la ciudad de Cuenca

\begin{tabular}{|c|c|c|c|c|c|}
\hline Establecimiento & Categoría & $\begin{array}{c}\text { Misión } \\
\text { y } \\
\text { Visión }\end{array}$ & $\begin{array}{c}\text { Política de } \\
\text { sostenibilidad }\end{array}$ & Planificación & $\begin{array}{c}\text { Evaluación de } \\
\text { cumplimiento } \\
\text { de acuerdo a } \\
\text { norma }\end{array}$ \\
\hline Hostal Casa Lloret & Primera & $\mathrm{Si}$ & $\mathrm{No}$ & $\mathrm{Si}$ & $80 \%$ \\
\hline Hotel Carvallo & Primera & $\mathrm{Si}$ & $\mathrm{No}$ & Parcialmente & $50 \%$ \\
\hline $\begin{array}{c}\text { Hotel Boutique } \\
\text { Mansión Alcázar }\end{array}$ & Lujo & $\mathrm{Si}$ & $\mathrm{Si}$ & $\mathrm{Si}$ & $100 \%$ \\
\hline Hotel Zahir $360^{\circ}$ & Primera & $\mathrm{Si}$ & No & Parcialmente & $50 \%$ \\
\hline Hotel Crespo & Primera & $\mathrm{No}$ & No & No & $0 \%$ \\
\hline Hostal Macondo & Segunda & $\mathrm{Si}$ & No & Parcialmente & $50 \%$ \\
\hline Hotel Rio Piedra & Segunda & $\mathrm{No}$ & No & No & $0 \%$ \\
\hline
\end{tabular}

Fuente: Elaboración propia a partir de los datos recolectados en las visitas realizadas a establecimientos.

Como se puede observar en la tabla 1 , tan solo un establecimiento cumple con todos los requisitos establecidos en este acápite. Por otra parte se cuenta con cuatro establecimientos de alojamiento (de diferente categoría) que cumplen parcialmente lo solicitado, dos de los cuales están catalogados como hoteles de primera de la ciudad de Cuenca. Por otra parte un sólo establecimiento no cumple con lo exigido por el sello, por tanto y de acuerdo a la presente investigación, existe un conocimiento sobre lo que constituye la sostenibilidad, sus herramientas y sus beneficios.

Por otra parte estos datos revelan que aunque mayoritariamente los establecimientos de alojamiento cumplen parcialmente con lo exigido en la norma, se observa que la tendencia de la mayoría de establecimientos es la de un enfoque más sostenible para la empresa, puesto que tan solo un establecimiento no cuenta con directrices que orienten a una gestión más efectiva y sostenible.

\subsection{Gestión de la calidad}

De acuerdo a la norma, la gestión de la calidad permite integrar la rentabilidad y la sostenibilidad de la empresa turística, pues permite organizar la empresa y su personal hacia la obtención de servicios y productos de calidad. Los criterios que establece este ámbito como requisitos indispensables para la acreditación de la norma son: Procesos y procedimientos, Administración y Dirección, Suministros y Proveedores, Monitoreo y acciones correctivas.

La Norma Smart Voyager establece el cumplimiento de los siguientes criterios:

Tabla 3 Criterios que establece la Norma Smart Voyager para la Gestión de la Calidad

\begin{tabular}{|c|c|c|c|}
\hline Criterio & Subcriterios & $\begin{array}{l}\text { Evidencias } \\
\text { requeridas }\end{array}$ & Evaluación \\
\hline $\begin{array}{l}\text { Procesos y } \\
\text { procedimien- } \\
\text { tos }\end{array}$ & $\begin{array}{c}\text { Programa de } \\
\text { trabajo }\end{array}$ & $\begin{array}{l}\text { - Manual de procesos } \\
\text { y procedimientos de } \\
\text { la empresa. } \\
\text { - Organigrama } \\
\text { - Cronograma de tra- } \\
\text { bajo } \\
\text { - Estandarización } \\
\text { de informes para } \\
\text { cumplimiento de } \\
\text { actividades }\end{array}$ & $25 \%$ \\
\hline
\end{tabular}


Tabla 3 Criterios que establece la Norma Smart Voyager para la Gestión de la Calidad (Continuación)

\begin{tabular}{|c|c|c|c|}
\hline Criterio & Subcriterios & $\begin{array}{l}\text { Evidencias } \\
\text { requeridas }\end{array}$ & Evaluación \\
\hline $\begin{array}{l}\text { Procesos y } \\
\text { procedimien- } \\
\text { tos }\end{array}$ & $\begin{array}{l}\text { Programa de } \\
\text { trabajo }\end{array}$ & $\begin{array}{l}\text { - Manual de procesos } \\
\text { y procedimientos de } \\
\text { la empresa. } \\
\text { - Organigrama } \\
\text { - Cronograma de tra- } \\
\text { bajo } \\
\text { - Estandarización } \\
\text { de informes para } \\
\text { cumplimiento de } \\
\text { actividades }\end{array}$ & $25 \%$ \\
\hline $\begin{array}{l}\text { Administracion } \\
\mathrm{y} \text { direccion }\end{array}$ & $\begin{array}{l}\text { Plan integral } \\
\text { de la empresa }\end{array}$ & $\begin{array}{l}\text { - Plan de trabajo para } \\
\text { cada área. }\end{array}$ & $25 \%$ \\
\hline $\begin{array}{l}\text { Suministros y } \\
\text { proveedores }\end{array}$ & $\begin{array}{c}\text { Manual de } \\
\text { suministros y } \\
\text { proveedores de } \\
\text { la empresa }\end{array}$ & $\begin{array}{l}\text { - Establecer con pro- } \\
\text { veedores las políticas } \\
\text { de pago y entrega de } \\
\text { mercadería. } \\
\text { - Cronograma de pedi- } \\
\text { dos por proveedor } \\
\text { - Política para la selec- } \\
\text { ción e proveedores. }\end{array}$ & $25 \%$ \\
\hline $\begin{array}{l}\text { Monitoreo y } \\
\text { acciones } \\
\text { correctivas }\end{array}$ & $\begin{array}{l}\text { Programa de } \\
\text { monitoreo y } \\
\text { acciones } \\
\text { correctivas }\end{array}$ & $\begin{array}{l}\text { - Seleccionar los servi- } \\
\text { cios a ser monitorea- } \\
\text { dos para lograr la ca- } \\
\text { lidad. } \\
\text { - Determinar porcenta- } \\
\text { je de cumplimiento } \\
\text { (calidad y cantidad) } \\
\text { de cada proceso y } \\
\text { procedimiento. } \\
\text { - Elaborar el cronogra- } \\
\text { ma de monitoreo. } \\
\text { - Definir acciones con- } \\
\text { cretas a los resulta- } \\
\text { dos de monitoreo }\end{array}$ & $25 \%$ \\
\hline
\end{tabular}

Fuente: Guía de Buenas Prácticas para Turismo Sostenible

Tabla 4 Gestión de la Calidad en establecimientos de alojamiento de la ciudad de Cuenca

\begin{tabular}{|c|c|c|c|c|c|}
\hline Establecimiento & $\begin{array}{c}\text { Procesos y } \\
\text { procedi- } \\
\text { mientos }\end{array}$ & $\begin{array}{c}\text { Administración } \\
\text { y dirección }\end{array}$ & $\begin{array}{c}\text { Suministros } \\
\text { y provee- } \\
\text { dores }\end{array}$ & $\begin{array}{c}\text { Monitoreo } \\
\text { y acciones } \\
\text { correctivas }\end{array}$ & $\begin{array}{c}\text { Evaluación de } \\
\text { cumplimiento } \\
\text { de acuerdo a } \\
\text { norma }\end{array}$ \\
\hline $\begin{array}{c}\text { Hostal Casa } \\
\text { Lloret }\end{array}$ & $\mathrm{No}$ & $\mathrm{Si}$ & $\mathrm{Si}$ & $\mathrm{Si}$ & $75 \%$ \\
\hline Hotel Carvallo & $\mathrm{Si}$ & $\mathrm{Si}$ & $\mathrm{Si}$ & $\mathrm{Si}$ & $100 \%$ \\
\hline $\begin{array}{c}\text { Hotel Boutique } \\
\text { Mansión Alcázar }\end{array}$ & $\mathrm{Si}$ & $\mathrm{Si}$ & $\mathrm{Si}$ & $\mathrm{Si}$ & $100 \%$ \\
\hline Hotel Zahir $360^{\circ}$ & $\mathrm{Si}$ & $\mathrm{Si}$ & $\mathrm{Si}$ & $\mathrm{Si}$ & $100 \%$ \\
\hline Hotel Crespo & Parcialmente & Parcialmente & $\mathrm{Si}$ & $\mathrm{No}$ & $50 \%$ \\
\hline Hostal Macondo & Parcialmente & $\mathrm{No}$ & Parcialmente & $\mathrm{No}$ & $25 \%$ \\
\hline Hotel Rio Piedra & Parcialmente & $\mathrm{No}$ & Parcialmente & $\mathrm{No}$ & $25 \%$ \\
\hline
\end{tabular}

Fuente: Elaboración propia a partir de los datos recolectados en las visitas realizadas a establecimientos.

De acuerdo a los resultados obtenidos presentados en la tabla 4, tres establecimientos (hoteles) cumplen con lo establecido en la norma, y cuatro establecimientos lo hacen de forma parcial. El área de mayor cumplimiento en este criterio, es la de suministros y proveedores, pues en la ciudad existen pocas empresas que se dedican al comercio para el insumo de productos de limpieza para establecimientos de alojamiento; otro dato relevante es el ámbito de la adquisición de alimentos, pues la gran mayoría de establecimientos, prefiere el servicio de empresas grandes como Supermaxi, por los plazos en cuento a pagos y precios al mayoreo, hecho que no se puede evidenciar en otros establecimientos de expendio masivos de la ciudad.

Las empresas de alojamiento de forma general, no cuentan con un sistema de monitoreo y control, pues gran parte de ellas, son empresas de carácter familiar, donde el aspecto empresarial es manejado de forma empírica o poco técnica y por ello no dedican sus esfuerzos al cumplimiento de este criterio.

Otro dato de gran importancia que muestran estos resultados, es que el aspecto que también refleja un poco cumplimiento es el de procesos y procedimientos, pues al ser empresas en su gran mayoría pequeñas, existe poco personal que desempeña más de una función dentro del establecimiento, y por ello no consideran relevante el contar con procesos.

\subsection{Gestión de Recursos Humanos}

Identificado como el recurso más importante de cualquier empresa, especialmente en aquellas dedicadas a la prestación de servicios, como lo es el sector turístico, este recurso está constituido por todos los trabajadores de la empresa, que se destacan por sus capacidades, conocimientos, experiencias e intereses que denotan en cumplimiento de sus actividades.

La Norma Smart Voyager estipula el cumplimiento de los siguientes criterios:

Tabla 5 Criterios para la Gestión de Recursos Humanos

\begin{tabular}{|c|c|c|c|}
\hline Criterio & Subcriterios & $\begin{array}{c}\text { Evidencias } \\
\text { requeridas }\end{array}$ & Evaluación \\
\hline $\begin{array}{c}\text { Manuales de } \\
\text { puestos y pro- } \\
\text { cedimientos }\end{array}$ & $\begin{array}{c}\text { Organigrama y } \\
\text { manual de } \\
\text { procesos y } \\
\text { puestos de } \\
\text { trabajo }\end{array}$ & $\begin{array}{c}\text { - Manual de puestos y } \\
\text { procedimientos. }\end{array}$ & $50 \%$ \\
\hline $\begin{array}{c}\text { Capacitación } \\
\text { al personal }\end{array}$ & $\begin{array}{c}\text { Plan integrado } \\
\text { de } \\
\text { capacitación } \\
\text { de la empresa }\end{array}$ & $\begin{array}{c}\text { Elaborar programas } \\
\text { de capacitación por } \\
\text { áreas Organigrama de ca- } \\
\text { pacitación }\end{array}$ & $50 \%$ \\
\hline
\end{tabular}

Fuente: Guía de Buenas Prácticas para Turismo Sostenible

Tabla 6 Gestión de Recursos Humanos en establecimientos de alojamiento de la ciudad de Cuenca

\begin{tabular}{|c|c|c|c|}
\hline Establecimiento & $\begin{array}{c}\text { Manuales de } \\
\text { puestos y } \\
\text { procedimientos }\end{array}$ & $\begin{array}{c}\text { Capacitación al } \\
\text { personal }\end{array}$ & $\begin{array}{c}\text { Evaluación de } \\
\text { cumplimiento de acuerdo } \\
\text { a norma }\end{array}$ \\
\hline Hostal Casa Lloret & No & No & 0 \\
\hline Hotel Carvallo & Parcialmente & Parcialmente & $50 \%$ \\
\hline $\begin{array}{c}\text { Hotel Boutique } \\
\text { Mansión Alcázar }\end{array}$ & $\mathrm{Si}$ & $\mathrm{Si}$ & $100 \%$ \\
\hline Hotel Zahir $360^{\circ}$ & $\mathrm{Si}$ & Parcialmente & $75 \%$ \\
\hline Hotel Crespo & No & No & $0 \%$ \\
\hline Hostal Macondo & Parcialmente & Parcialmente & $50 \%$ \\
\hline Hotel Rio Piedra & No & Parcialmente & $25 \%$ \\
\hline
\end{tabular}

Fuente: Propia a partir de los datos obtenidos de las visitas de campo y evidencias presentadas por los establecimientos. 
Es evidente que el aspecto en el cual los establecimientos de alojamiento muestran menor interés es en la capacitación al personal, este hecho puede deducirse al temor que muchos empresarios turísticos mantienen, que tras capacitar a sus empleados, estos opten por buscar un mejor empleo o mejorar su salario en otra empresa. Otra de las razones que inciden en este aspecto, son los costos que implican los cursos de capacitación, que muchas empresas no pueden asumirlo en las actuales circunstancias que experimenta el sector alojamiento en la ciudad; instituciones como la Cámara de Turismo del Azuay, AHOTEC y otras entidades no gubernamentales, ofertan permanentemente con programas de capacitación gratuitos, los cuales lamentablemente no son lo suficientemente promocionados en el sector.

\subsection{Gestión Financiera Contable}

Una efectiva gestión financiera permite analizar la dinámica del flujo de dinero en una empresa determinada, su efectiva administración permitirá contar con información real y útil para la toma de decisiones económicas.

La Norma Smart Voyager establece que la acreditación de este criterio obedece al cumplimiento de:

Tabla 7 Criterios para la Gestión Financiera Contable

\begin{tabular}{|c|c|c|c|}
\hline Criterio & Subcriterios & $\begin{array}{c}\text { Evidencias } \\
\text { requeridas }\end{array}$ & Evaluación \\
\hline $\begin{array}{c}\text { Sistema } \\
\text { financiero } \\
\text { contable }\end{array}$ & $\begin{array}{c}\text { Manual de } \\
\text { procedimien- } \\
\text { tos financieros } \\
\text { y contables }\end{array}$ & $\begin{array}{c}\text { - Informes de audito- } \\
\text { rías externas de las } \\
\text { áreas financieras. }\end{array}$ & $50 \%$ \\
\hline Presupuestos & $\begin{array}{c}\text { Presupuesto } \\
\text { maestro } \\
\text { intermedios } \\
\text { Presupuestos operati- } \\
\text { vos } \\
\text { Presupuestos de in- } \\
\text { versiones }\end{array}$ & $50 \%$ \\
\hline
\end{tabular}

Fuente: Guía de Buenas Prácticas para Turismo Sostenible

Tabla 8 Gestión Financiera Contable en establecimientos de alojamiento de la ciudad de Cuenca

\begin{tabular}{|c|c|c|c|}
\hline Establecimiento & $\begin{array}{c}\text { Sistema financiero } \\
\text { contable }\end{array}$ & Presupuestos & $\begin{array}{c}\text { Evaluación de } \\
\text { cumplimiento de acuerdo } \\
\text { a norma }\end{array}$ \\
\hline Hostal Casa Lloret & Parcialmente & Parcialmente & $60 \%$ \\
\hline Hotel Carvallo & $\mathrm{Si}$ & $\mathrm{Si}$ & $100 \%$ \\
\hline $\begin{array}{c}\text { Hotel Boutique } \\
\text { Mansión Alcázar }\end{array}$ & $\mathrm{Si}$ & $\mathrm{Si}$ & $100 \%$ \\
\hline Hotel Zahir $360^{\circ}$ & $\mathrm{Si}$ & $\mathrm{Si}$ & $100 \%$ \\
\hline Hotel Crespo & Parcialmente & Parcialmente & $60 \%$ \\
\hline Hostal Macondo & Parcialmente & Parcialmente & $60 \%$ \\
\hline Hotel Rio Piedra & Parcialmente & No & $25 \%$ \\
\hline
\end{tabular}

Fuente: Propia a partir de los datos obtenidos de las visitas de campo y evidencias presentadas por los establecimientos.

Los datos presentados en la tabla 8, demuestran que la mayoría de establecimientos si bien cuentan con sistemas financieros contables, éstos no son actualizados acorde a las nuevas exigencias que exige el sector, como sistemas contables específicos para establecimientos de alojamiento, lamentablemente muchos establecimientos no cuentan con manuales de procedimientos financieros y contables, aunque si presentan informes de auditorías efectuadas. En lo referente a presupuestos, éstos son llevados digitalmente y no en sistemas especializados acordes al sector turístico.

\subsection{Gestión de seguridad}

Considerada como la base medular de una empresa, mediante la cual se estipulan los elementos laborales, de higiene y salubridad, prevención de actos delictivos y los accidentes en las operaciones turísticas, una efectiva gestión de la seguridad puede minimizar los riesgos, accidentes e incidentes y las enfermedades que puedan presentarse en todas las acciones y servicios empresariales.

De acuerdo a la Norma Smart Voyager, la gestión de seguridad involucra el cumplimiento de los siguientes criterios:

Tabla 9 Criterios para la Gestión de la seguridad

\begin{tabular}{|c|c|c|c|}
\hline Criterio & Subcriterios & $\begin{array}{l}\text { Evidencias } \\
\text { requeridas }\end{array}$ & Evaluación \\
\hline $\begin{array}{l}\text { Gestión de } \\
\text { seguridad } \\
\text { empresarial }\end{array}$ & $\begin{array}{l}\text { Programa de } \\
\text { gestión de } \\
\text { seguridad }\end{array}$ & $\begin{array}{l}\text { - Manual de riesgos de } \\
\text { la empresa. } \\
\text { - Plan de contingencia } \\
\text { en caso de catástrofes } \\
\text { o riesgos emergentes. } \\
\text { - Planes preventivos de } \\
\text { riesgos } \\
\text { - Auditorías de riesgos } \\
\text { - Matriz de riesgos }\end{array}$ & $100 \%$ \\
\hline
\end{tabular}

Fuente: Guía de Buenas Prácticas para Turismo Sostenible

Tabla 10 Gestión de Seguridad en establecimientos de alojamiento de la ciudad de Cuenca

\begin{tabular}{|c|c|c|}
\hline Establecimiento & $\begin{array}{c}\text { Gestión de } \\
\text { seguridad } \\
\text { empresarial }\end{array}$ & $\begin{array}{c}\text { Evaluación de } \\
\text { cumplimiento } \\
\text { de acuerdo a } \\
\text { norma }\end{array}$ \\
\hline Hostal Casa Lloret & Parcialmente & $70 \%$ \\
\hline Hotel Carvallo & Parcialmente & $50 \%$ \\
\hline Hotel Boutique Mansion Alcazar & $\mathrm{Si}$ & $100 \%$ \\
\hline Hotel Zahir $360^{\circ}$ & $\mathrm{Si}$ & $100 \%$ \\
\hline Hotel Crespo & Parcialmente & $75 \%$ \\
\hline Hostal Macondo & Parcialmente & $50 \%$ \\
\hline Hotel Rio Piedra & Parcialmente & $50 \%$ \\
\hline
\end{tabular}

Fuente: Propia a partir de los datos obtenidos de las visitas de campo y evidencias presentadas por los establecimientos.

El tema de la seguridad constituye uno de los elementos más vulnerables en la gestión hotelera en la ciudad de Cuenca, pues gran parte de estos establecimientos se ubican en casas patrimoniales. En el caso de los hoteles seleccionados, dos hoteles se ubican en este tipo de viviendas, así como una hostal. Lamentablemente los permisos de funcionamiento que exige el municipio no establecen la implementación de salidas de emergencia o contar con planes de seguridad, por lo tanto gran parte de estos establecimientos aunque son conscientes de los potenciales 
riesgos que implican estas edificaciones, no constituye un tema prioritario para su gestión. Entre las evidencias que se ha podido recabar en esta investigación, la presencia de manuales de riesgos es el aspecto en el que mayoritariamente cumplen estos establecimientos, los cuales presentan los potenciales riesgos y capacitaciones para el personal, sin embargo no estipula ningún plan preventivo o un plan de contingencia para casos de catástrofes.

\subsection{Gestión de la comunicación}

Constituyen todas las acciones encaminadas a la difusión y conocimiento de los productos y servicios que una empresa determinada ofrece; para ello es preciso planificar y enfocarse de manera estratégica a los potenciales clientes de manera efectiva, a través del uso de medios estipulados para ello.

Tabla 11 Criterios para la Gestión de la Comunicación

\begin{tabular}{|c|c|c|c|}
\hline Criterio & Subcriterios & $\begin{array}{c}\text { Evidencias } \\
\text { requeridas }\end{array}$ & Evaluación \\
\hline $\begin{array}{c}\text { Mercadeo y } \\
\text { comunicación }\end{array}$ & $\begin{array}{c}\text { Plan de } \\
\text { comunicación } \\
\text { empresarial } \\
\text { ducto y sus caracte- } \\
\text { rísticas. } \\
\text { - Política de sostenibi- } \\
\text { lidad y comunicación }\end{array}$ & $50 \%$ \\
\hline Mercadeo & Plan de & $\begin{array}{l}\text { - Cronograma de venta } \\
\text { y comercialización. } \\
\text { Programa de evalua- } \\
\text { ción y medición de } \\
\text { resultados de accio- } \\
\text { nes de mercadeo y } \\
\text { publicidad. }\end{array}$ & $50 \%$ \\
\hline
\end{tabular}

Fuente: Guía de Buenas Prácticas para Turismo Sostenible

La comunicación y el mercadeo constituyen herramientas indispensables para el desarrollo y crecimiento de una empresa turística; como se puede evidenciar, las empresas seleccionadas cumplen parcialmente con este requerimiento al consolidar sus estrategias de comunicación mediante redes sociales y publicaciones en revistas especializadas. Sin embargo no cuentan con un programa de evaluación y medición de resultados sobre las acciones de mercadeo y publicidad, así como con políticas de sostenibilidad y comunicación. Las razones por las cuales no se ha invertido tiempo en el desarrollo de estas herramientas, radica una vez más al hecho de que gran parte de estos establecimientos constituyen empresas familiares, donde no se evidencia una formación técnica en el área de la hospitalidad y por tanto un desconocimiento de estas acciones. Por otra parte las ventas se canalizan de forma digital a través de páginas web y no se generan alianzas estratégicas con operadores de turismo u otras empresas de intermediación.
Tabla 12 Gestión Financiera Contable en establecimientos de alojamiento de la ciudad de Cuenca

\begin{tabular}{|c|c|c|c|}
\hline Establecimiento & $\begin{array}{c}\text { Mercadeo y } \\
\text { comunicación }\end{array}$ & Mercadeo & $\begin{array}{c}\text { Evaluación de } \\
\text { cumplimiento de acuerdo } \\
\text { a norma }\end{array}$ \\
\hline Hostal Casa Lloret & $\mathrm{Si}$ & $\mathrm{Si}$ & $100 \%$ \\
\hline Hotel Carvallo & Parcialmente & Parcialmente & $50 \%$ \\
\hline $\begin{array}{c}\text { Hotel Boutique } \\
\text { Mansión Alcázar }\end{array}$ & Parcialmente & Parcialmente & $75 \%$ \\
\hline Hotel Zahir $360^{\circ}$ & $\mathrm{Si}$ & $\mathrm{Si}$ & $100 \%$ \\
\hline Hotel Crespo & Parcialmente & Parcialmente & $75 \%$ \\
\hline Hostal Macondo & Parcialmente & Parcialmente & $75 \%$ \\
\hline Hotel Rio Piedra & Parcialmente & No & $35 \%$ \\
\hline
\end{tabular}

Fuente: Propia a partir de los datos obtenidos de las visitas de campo y evidencias presentadas por los establecimientos.

\section{Discusión de resultados}

Los resultados del estudio del ámbito empresarial de la Norma Smart Voyager empleada por la organización Conservación y Desarrollo, muestran que en la ciudad de Cuenca, los establecimientos de alojamiento tienen claro sus objetivos, los cuales se ven plasmados en su misión y visión, aunque gran parte de ellos no cuenta con un plan estratégico que oriente sus acciones, otro aspecto relevante que muestra este estudio, es el hecho de no contar con organigramas o manuales de puestos y procesos, pues como se ha indicado anteriormente se trata de empresas familiares que no acogen un gran número de personas, quienes a su vez desempeñan varias funciones a la vez. La política de sostenibilidad por tanto no es un factor determinante en las empresas de alojamiento, aunque gran parte de ellas conoce o mantiene alguna referencia sobre este concepto.

Por su parte estos establecimientos tienen definidos y organizados a las empresas que les sirven como proveedoras de los insumos necesarios para su operación, sin embargo, estos proveedores no so necesariamente locales, pues gran parte de las empresas de alojamiento buscan facilidades de pago como requisito previo para definir sus alianzas. En lo referente a recursos humanos, si bien la oferta de profesionales en el área de la hospitalidad se ha incrementado en la ciudad; los establecimientos de alojamiento no consideran una prioridad el establecer programas de capacitación para sus empleados, pues como se indicó anteriormente, los gerentes consideran un alto riesgo el capacitar a su personal, pues constituye una oportunidad para el empleado para cambiar de trabajo con mejores posibilidades remunerativas.

La gestión financiera - contable constituyó el área más sensible para el desarrollo de este estudio, pues muchas de las empresas visitadas, no cuentan con suficiente información visible para su análisis, sin embargo se puede deducir, que los establecimientos manejan su sistema financiero contable, con programas contables generales, no aplicables al área de la hospitalidad, y en su gran mayoría depositan esta tarea a un contador externo a la empresa.

Otro aspecto que llama mucho la atención, es la falta de interés que muchos de los establecimientos de alojamiento muestran respecto de la seguridad, pues como se mencionó anteriormente se trata de viviendas de la época 
Republicana que fueron adaptadas para funcionar como empresas de alojamiento, por tanto no existen planes o programas de seguridad, prevención de riesgos o cualquier otra documentación que permita actuar en caso de situaciones extremas.

El último criterio analizado en esta investigación es la comunicación y el mercadeo, actividades básicas y necesarias para la operación de la empresa, los resultados demuestran que el uso de tecnologías de la información, redes sociales y medios digitales en general son la herramienta más utilizada para llevar a cabo estas actividades, sin embargo se evidencia la falta de alianzas estratégicas con sectores vinculados a la hospitalidad en la ciudad de Cuenca.

\section{Conclusiones}

La aplicación de buenas prácticas para turismo sostenible, demanda el cumplimiento de varios requisitos que en gran parte implican un cambio importante para la empresa; pero por sobretodo un cambio en la visión de quienes están al frente de las mismas. La Norma Smart Voyager define seis criterios que son la base sobre la cual se debe desarrollar el ámbito empresarial del establecimiento, la presente investigación buscó recabar toda evidencia plasmada en este documento para evaluar su porcentaje de cumplimiento, sin embargo se observa que aunque no se cuente con la totalidad de los requisitos solicitados, las empresas cuentan con suficientes herramientas para avanzar hacia un desarrollo más sostenible.

En la ciudad de Cuenca las empresas certificadas en calidad son pocas, y dentro del área de la sostenibilidad no existen, por tanto este estudio demuestra que se está en buen camino, independientemente de su categoría, los establecimientos de alojamiento pueden iniciar con procesos de certificación en esta área en un futuro no muy lejano.

El contar con establecimientos certificados en sostenibilidad, constituiría un atractivo más para la ciudad de Cuenca, considerando las nuevas tendencias en alojamiento la actitud que demuestran los turistas actualmente, se hace necesario tomar medidas a nivel gubernamental para incentivar este tipo de actividades.

\section{Referencias Bibliográficas}

Ayaviri, V., Quispe, G., y Sánchez, P. (2017). Estimación de la demanda del turismo en la comunidad andina de naciones. Revista Espacios, 2, 24-38.

Ayuso, S. (2008). Gestión sostenible en la industria turística (2008th ed.). Barcelona.

Boullón, R. (2006). Planificación del espacio turístico. Madrid.
Campodónico, R. (2016). Turismo: de la movilidad al espacio (Vol. 24).

De las Heras, M. (2008). Manual de ecoturismo. Madrid.

Fundación Municipal de Turismo para Cuenca y Ministerio de Turismo. (2011). Plan estratégico de desarrollo turístico del cantón cuenca y su Área de influencia (2011th ed.). Cuenca: Fundación Municipal de Turism para Cuenca..

Hoteles Sostenibles. (2016). Sostenibilidad en el mundo de la hospitalidad.

Organización Mundial del Turismo. (2013). Notas metodológicas de la base de datos de estadisticas de turismo (2013th ed.). Madrid.

Organización Mundial del Turismo. (2017). Catastro hotelero (2017th ed.). Cuenca: Editorial Ministerio del Turismo.

Pulido Fernández, J. I. (2005). Criterios para una política turística sostenible en los parques naturales de andalucía (Vol. 24).

Pulido Fernández, J. I. (2006). El medio ambiente en la política turística española (Vol. 7) (n. $\left.{ }^{\circ} 22\right)$.

Pulido Fernández, J. I. (2015). Criterios para una política turística sostenible en los parques naturales de andalucía (Vol. 23).

Rainfores Alliance. (2004). Guía de buenas prácticas para turismo sostenible. Belize: Rainfores Alliance.

Santiago Romero, N., y Alvarez, G. (2017). Actualidad y proyecciones de desarrollo del turismo internacional en ecuador. Revista de Ciencia, Tecnología e Innovación, 3, 276-287.

Tour Operator Initiative. (2013). Porqué hablar de sostenibilidad en hoteles (2013th ed.). Bogotá.

TripAdvisor. (2013). Nuevas tendencias en los viajeros del siglo xxi. (Vol. 121).

Recibido: 30 de junio de 2018

Aceptado: 3 de septiembre de 2018 